\title{
Dissecção espontânea da aorta abdominal infrarrenal
}

\section{Spontaneous dissection of the infrarenal aorta}

\author{
Otacílio de Camargo Junior', Antonio Cláudio Guedes Chrispim², Claudio Roberto Cabrini Simões², \\ Márcia Fayad Marcondes², Guilherme Camargo Gonçalves de Abreu², Guilherme Vieira Meirelles², \\ Kelly Cristina de Moraes ${ }^{3}$, Marcio Villar de Freitas ${ }^{3}$, Marivan Pedra Araújo ${ }^{3}$, Gustavo Braga Murta ${ }^{3}$
}

\section{Resumo}

A rotura da camada íntima que marca o início da dissecção aórtica se origina na maioria dos casos na aorta torácica, sendo rara a dissecção espontânea da aorta abdominal infra-renal. As três principais causas são: iatrogênica, traumática ou espontânea. A dor abdominal e a isquemia de membros são os sintomas mais comuns e um número significativo de pacientes e' assintomatico. O diagnóstico tem sido feito através de métodos de imagem como ultrassonografia, tomografia computadorizada, ressonância nuclear magnética e angiografia aliados ao alto índice de suspeição. Relatamos os casos de duas pacientes que apresentaram dissecção de aorta abdominal infrarrenal com quadro de dor abdominal súbita, sem sinais de irritação peritoneal com pulsos presentes e simétricos ao exame físico que deram entrada no pronto socorro do Hospital e Maternidade Celso Pierro da PUC Campinas e que foram tratadas pela equipe de Cirurgia Vascular.

As duas pacientes no momento do exame apresentavam-se hipertensas e ao ultrassom apresentavam alteração da conformidade da aorta abdominal que foram tratada s cirurgicamente. Uma paciente foi tratada cirurgicamente submetida a endarterectomia da placa dissecada da aorta abdominal infrarrenal de 2,2 cm de diâmetro e 2,0 cm de extensão. A outra paciente foi submetida a revascularização da aorta abdominal bi-iliaca com prótese de Dacron $16 \times$ $8 \mathrm{~mm}$ por apresentar disseccao da aorta abdominal distal.

As duas pacientes apresentaram boa evolução pos-operatoria tendo alta hospitalar em bom estado geral.

Descritores: dissecção; aorta abdominal; atherosclerosis.

\begin{abstract}
The rupture of the intimal layer marks the beginning of the aortic dissection, which usually happens in the thoracic aorta. The spontaneous dissection of the infrarenal aorta is rare. The main causes are: iatrogenic, traumatic and spontaneous. Abdominal pain and limb ischemia are the commonest symptoms, and some patients are asymptomatics. The diagnosis is made by ultrasound, computed tomography, nuclear magnetic resonance and angiography, with a high suspicious index. We describe two cases of spontaneous rupture of the infrarenal aorta that were treated surgically. We report two cases of patients who were admitted to the emergency room of Celso Pierro Hospital with infrarenal abdominal aortic dissection and were treated by the vascular surgery group. They have presented sudden abdominal pain, no signs of peritoneal irritation and pulses were presents and simmetrics in physical examination The two patients during the examination were hypertensive and the ultrasound showed abnormal compliance of the abdominal aorta who were treated surgically. One patient was treated surgically with dissected plaque endarterectomy of infrarenal abdominal aorta of $2.2 \mathrm{~cm}$ in diameter and $2.0 \mathrm{~cm}$ in length. The other patient underwent revascularization of the abdominal aorta with bi-iliac Dacron graft $16 \times 8 \mathrm{~mm}$ due to distal abdominal aortic dissection. Both patients had good postoperative evolution and they were discharged in good general condition.
\end{abstract}

Keywords: dissection; aorta, abdominal; atherosclerosis.

Trabalho Apresentado no IX Panamerican Congress on Vascular and Endovascular Surgery

Professor Adjunto, Chefe do Serviço de Angiologia e Cirurgia Vascular do Hospital e Maternidade Celso Pierro (HMCP), Pontifícia Universidade Católica (PUC-CAMPINAS), Campinas, SP, Brasil.

${ }^{2}$ Médico do Serviço de Angiologia e Cirurgia Vascular do Hospital e Maternidade Celso Pierro (HMCP), Pontifícia Universidade Católica (PUC-CAMPINAS), Campinas, SP, Brasil.

${ }^{3}$ Residente do Serviço de Angiologia e Cirurgia Vascular do Hospital e Maternidade Celso Pierro (HMCP), Pontifícia Universidade Católica (PUC-CAMPINAS), Campinas, SP, Brasil.

Fonte de financiamento: Não houve financiamento

Conflito de Interesse: Não Há

Submetido em: 11.4.2010. Aceito em: 25.6.2012.

J Vasc Bras. 2012;11(3):240-245. 


\section{Introdução}

A dissecção aórtica é a entidade clínica aguda grave mais comum a acometer a aorta, sendo uma vez e meia mais frequente que o aneurisma de aorta abdominal roto e, pelo menos, quatro vezes mais comum que o aneurisma torácico roto $^{1}$. A rotura da camada íntima que marca o início da dissecção aórtica se origina na maioria dos casos na aorta torácica, sendo rara a dissecção espontânea da aorta abdominal infrarrenal ${ }^{2,3}$. As três principais causas são: iatrogênica, traumática ou espontânea. A dor abdominal e a isquemia de membros inferiores são os sintomas mais comuns ${ }^{2}$ e um número significativo de pacientes e assintomático ${ }^{4}$.

A incidência de dissecção isolada da aorta abdominal é rara correspondendo a menos de $2 \%$ dos casos de dissecção de aorta. A dissecção da aorta ascendente corresponde a 70\%, a de aorta descendente a $20 \%$ e a de arco aórtico a $7 \%$ dos casos relatados na literatura ${ }^{5}$. Relatamos os casos de duas pacientes que apresentaram dissecção espontånea da aorta abdominal infrarrenal que foram tratadas cirurgicamente.

\section{Relato de caso}

\section{Caso 1}

$\mathrm{P}$ aciente de 67 anos, sexo feminino, hipertensa e dislipidêmica com história de dor abdominal súbita em mesogastro. Ao exame físico apresentava dor abdominal difusa à palpação sem sinais de irritação peritoneal, pulsos simétricos em membros superiores e inferiores. A pressão arterial era de $180 \times 100 \mathrm{mmHg}$. Ao ultra-som abdominal apresentava alteração na conformação da aorta abdominal. Solicitado aortografia abdominal (Figuras 1 e 2) angiotomografia de abdome (Figura 3) que evidenciaram dissecção de aorta abdominal infrarrenal de 2,2 cm de diâmetro e 2,0 cm de extensão. Submetida a endarterectomia de aorta abdominal infrarrenal (Figuras 4-7) evoluiu sem intercorrências e recebeu alta hospitalar no quinto dia de pós-operatório.

\section{Caso 2}

Paciente com 73 anos, sexo feminino, hipertensa, dislipidêmica e portadora de fibrilação atrial apresentou dor abdominal súbita localizada em região hipogástrica. Ao exame físico apresentava dor abdominal difusa à palpação, ausência de sinais de irritação peritoneal, pulsos presentes e simétricos em membros superiores e inferiores, pressão arterial de $190 \times 110 \mathrm{mmHg}$. Ao ultra-som abdominal apresentava alteração do diâmetro da aorta abdominal. Foi realizada uma

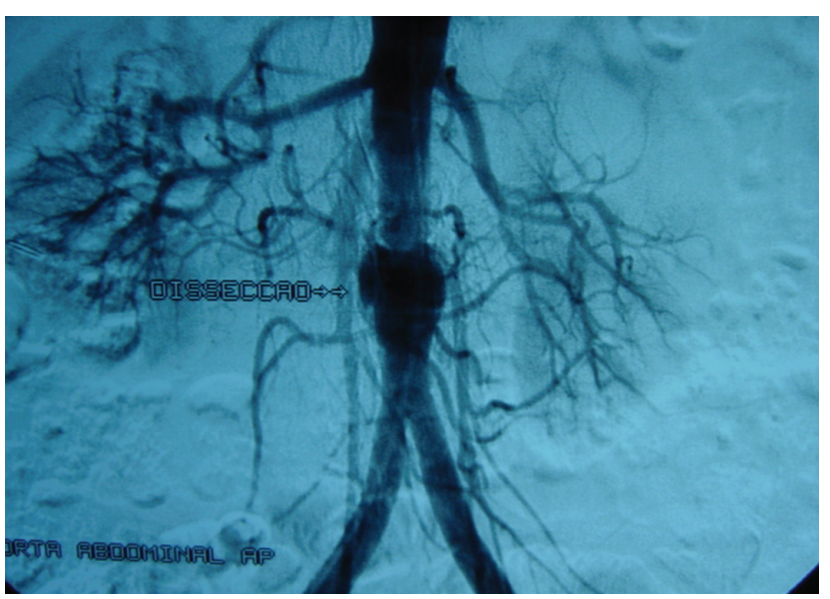

Figura 1. Arteriografia: dissecção de aorta abdominal (caso 1).

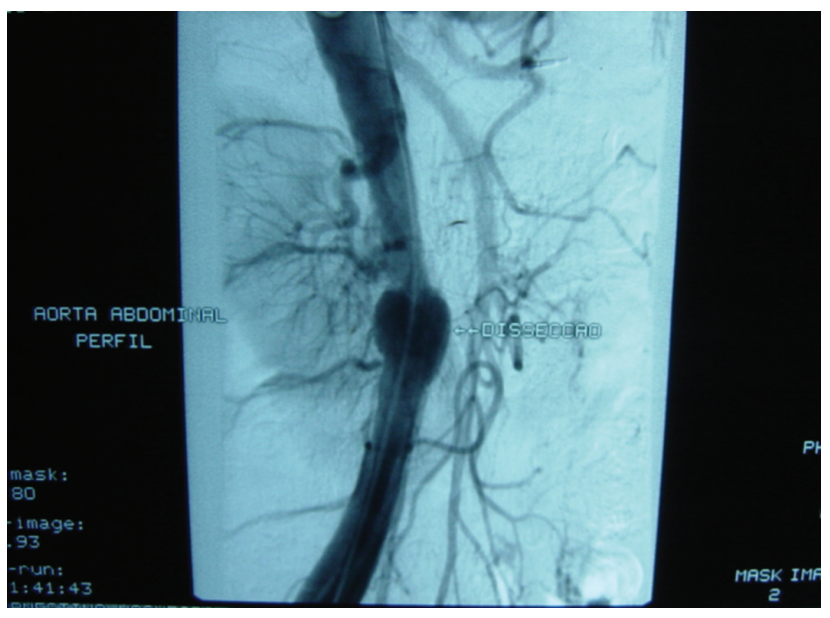

Figura 2. Arteriografia: dissecção de aorta abdominal (caso 1).

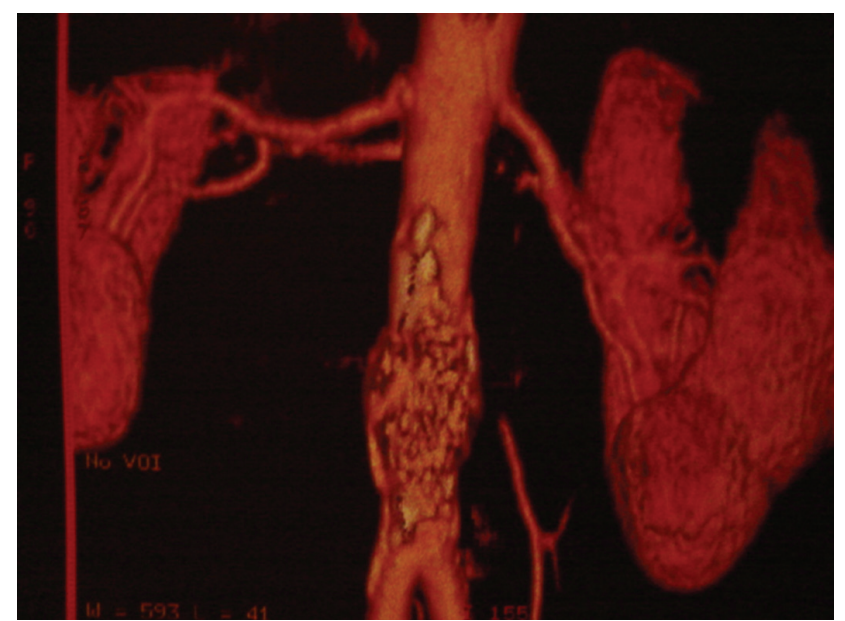

Figura 3. Angiotomografia: dissecção de aorta abdominal (caso 1). 


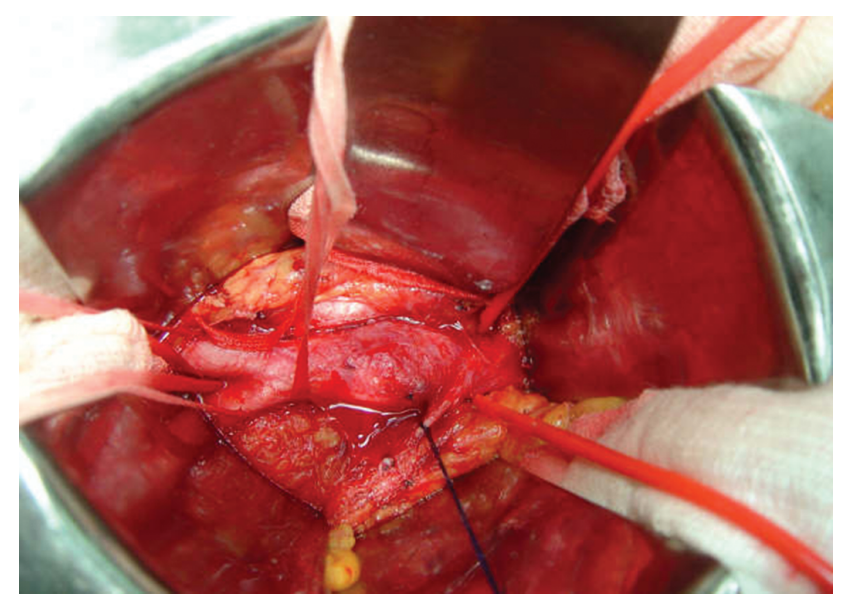

Figura 4.Intra-operatório: segnento aorto-ilíaco (caso 1).

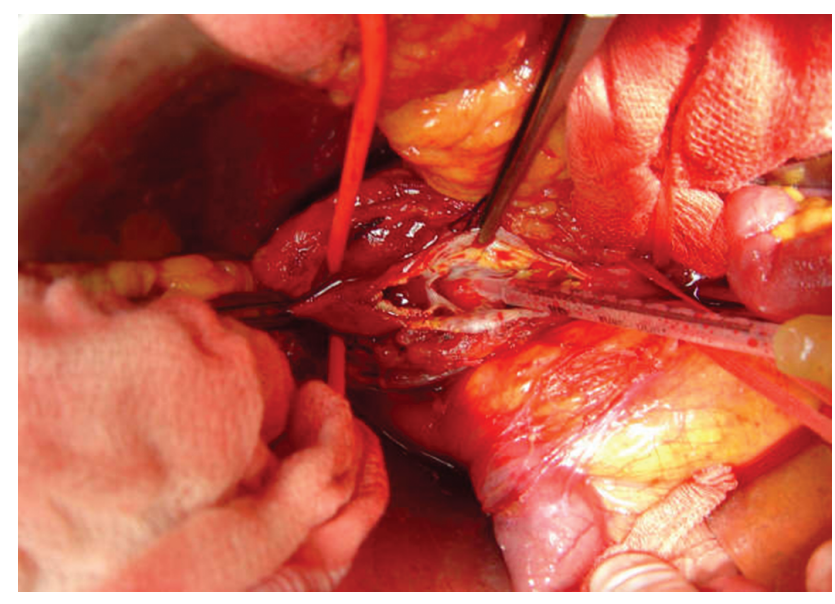

Figura 5. Intra-operatório: arteriotomia de aorta abdominal (caso 1).

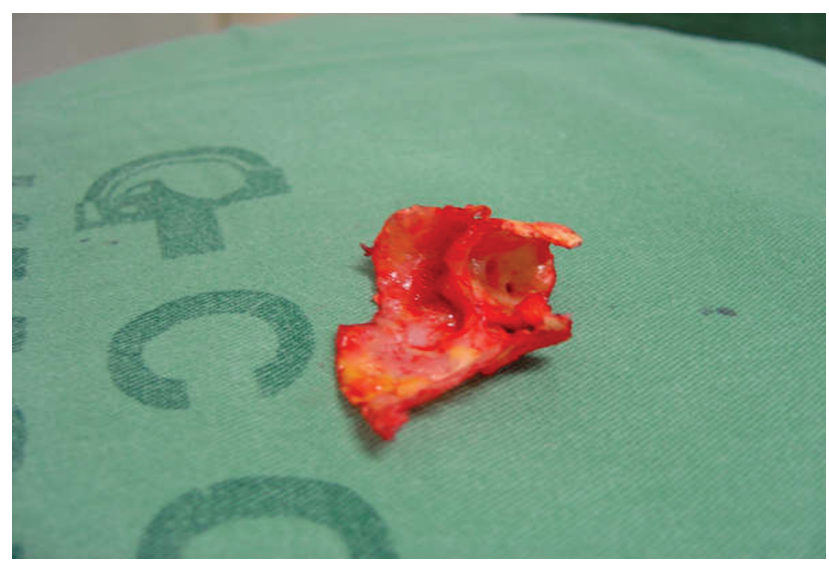

Figura 6. Placa de dissecção (caso 1).

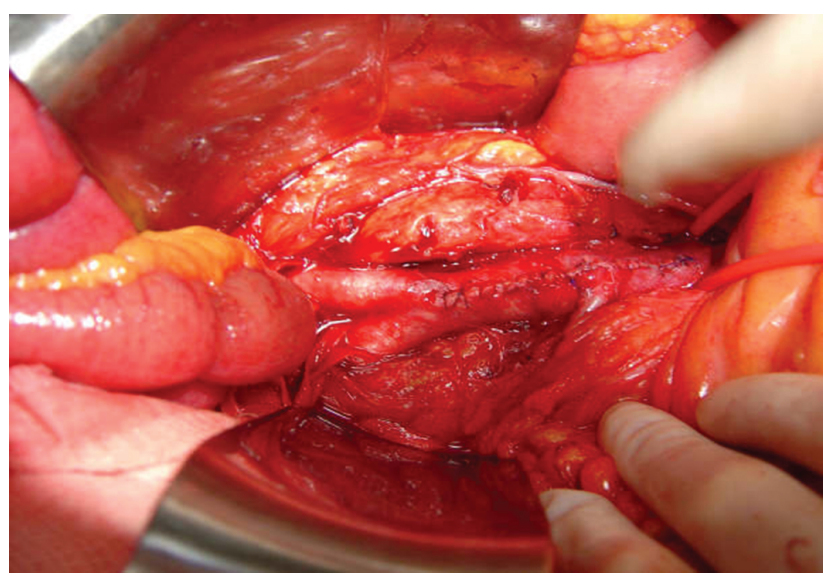

Figura 7. Pós endarterectomia (caso 1).

tomografia computadorizada que evidenciou aneurisma de aorta torácica ascendente (Figura 8) com diâmetro de 4,3 cm e dissecção de aorta abdominal terminal (Figuras 9 e 10) com $3,5 \mathrm{~cm}$ em seu maior diâmetro e $4,0 \mathrm{~cm}$ de extensão, aneurisma de artérias ilíacas comuns de 2,7 cm à direita e 2,0 cm à esquerda. Ao ultra-som Doppler arterial de membros inferiores apresentava ausência de dilatação aneurismática ou estenose significativa. Submetida à arteriografia que evidenciou aorta abdominal infra-renal e artérias ilíacas tortuosas e de calibre aumentado e dissecção infrarrenal com progressão para a artéria ilíaca comum direita (Figuras 11 e 12). Submetida à derivaçao aorto ilíaca comum esquerda e ilíaca externa direita. A paciente apresentou boa evolução e recebeu alta no quinto dia de pós-operatório.

\section{Discussão}

A dissecção espontânea da aorta abdominal isolada é um evento raro ${ }^{4-8}$ ocorre em $1,3 \%$ dos casos segundo dados do Registro Internacional de Dissecção Aórtica Aguda (International Registry of Acute Aortic Dissection: IRAAD) ${ }^{7}$. A experiência clínica dessa condição é baseada em relatos de casos e pequenas séries de casos, a maior delas é do IRAAD que consiste em 18 pacientes?

A idade média de apresentação é entre 50 e 60 anos $^{8}$. As dissecções espontâneas da aorta abdominal infrarrenal são classificadas clinicamente em três grupos: assintomático, cujo diagnóstico e realizado por exames de imagem, correspondendo por cerca de 25\% dos casos; grupo com dor (quase $60 \%$ dos casos), que se apresenta com quadro doloroso abdominal ou lombar; e grupo com isquemia dos membros inferiores, 


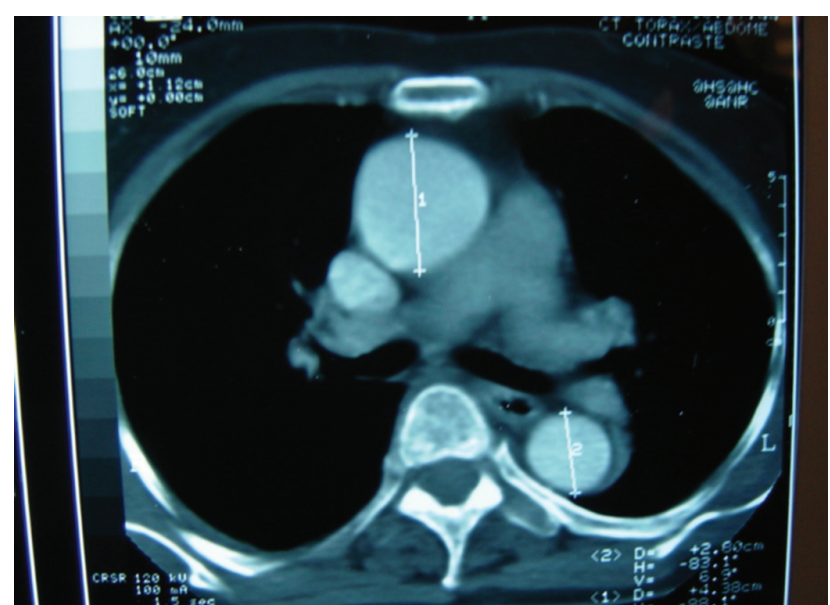

Figura 8. TC: aneurisma de aorta torácica (caso 2).

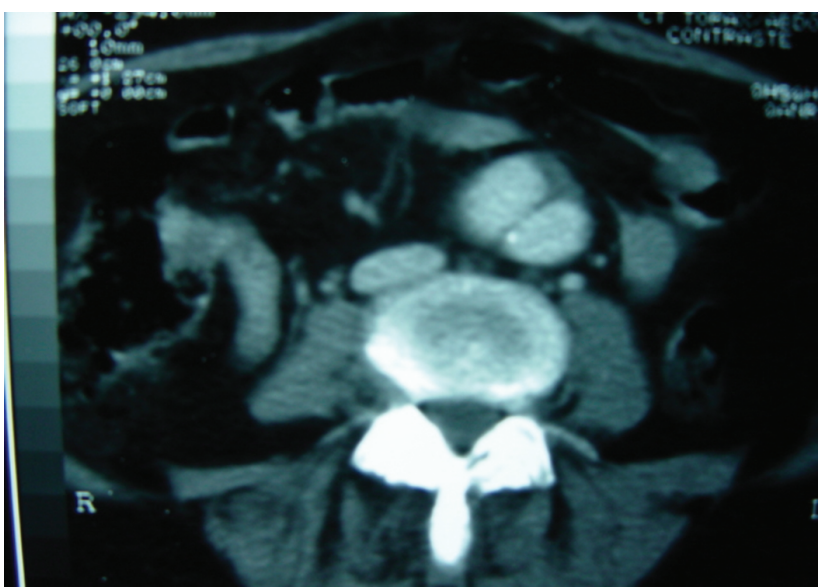

Figura 9. TC : dissecção da aorta abdominal (caso 2).

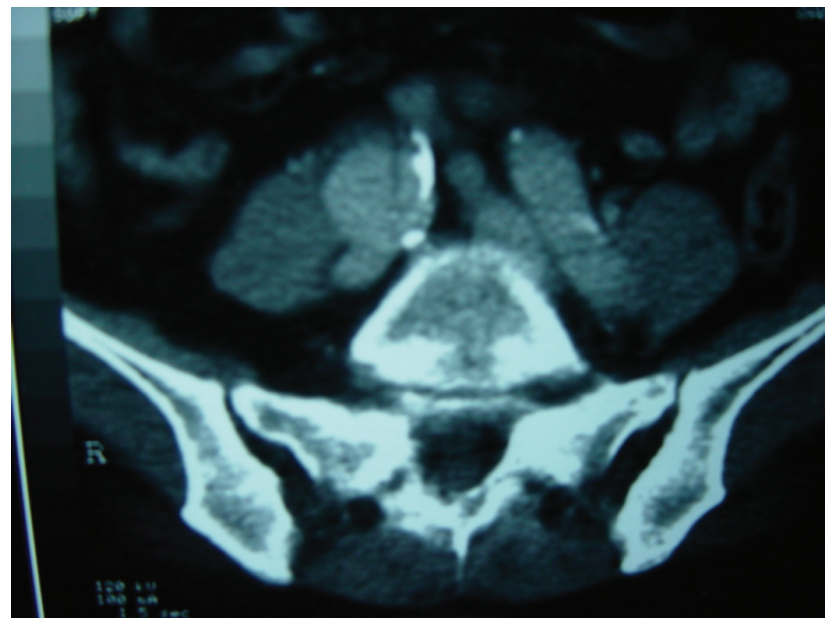

Figura 10. TC: dissecção da artéria ilíaca direita (caso 2). por oclusão arterial aguda ou por claudicação intermitente, atingindo cerca de $15 \%$ dos casos ${ }^{2}$.

Em uma revisão da literatura ${ }^{3}$ feita por Mozes e colegas foram encontrados 41 casos de dissecção espontânea da aorta abdominal infrarrenal (DEAAI). A idade média foi de 58 anos, $74 \%$ dos pacientes eram do sexo masculino e $62 \%$ tinham hipertensão. A dissecção ficou limitada à aorta abdominal infra-renal em $50 \%$ dos casos e estendeu-se às artérias ilíacas e femorais em $50 \%$.

Em outra revisão da literatura ${ }^{9}$, a rotura mostrou-se mais frequente (17\%) que os dados da literatura e o choque hemorrágico apresentou-se como sinal mais freqüente (66,6\%). A taxa de mortalidade relacionada à rotura da dissecção foi de $17,7 \%$.

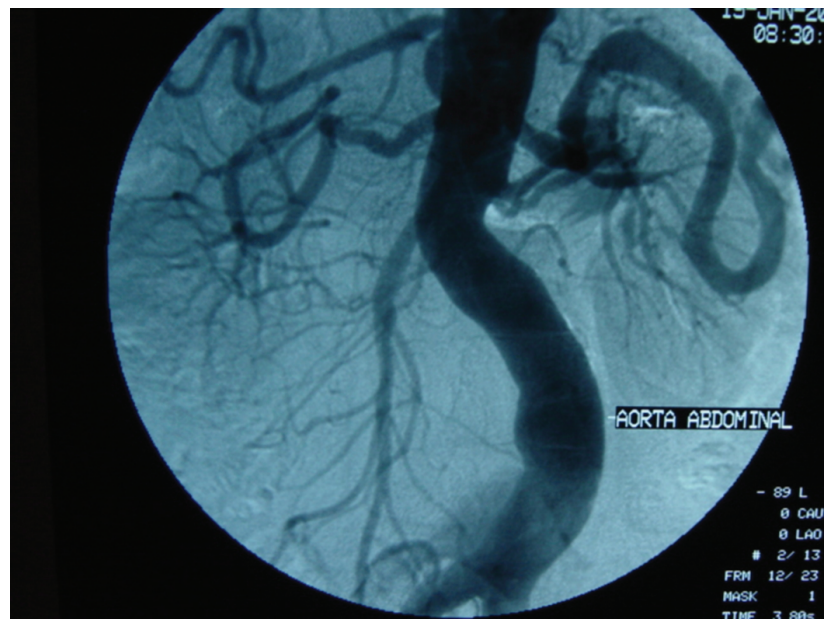

Figura 11. Arteriografia: dissecção de aorta abdominal (caso 2).

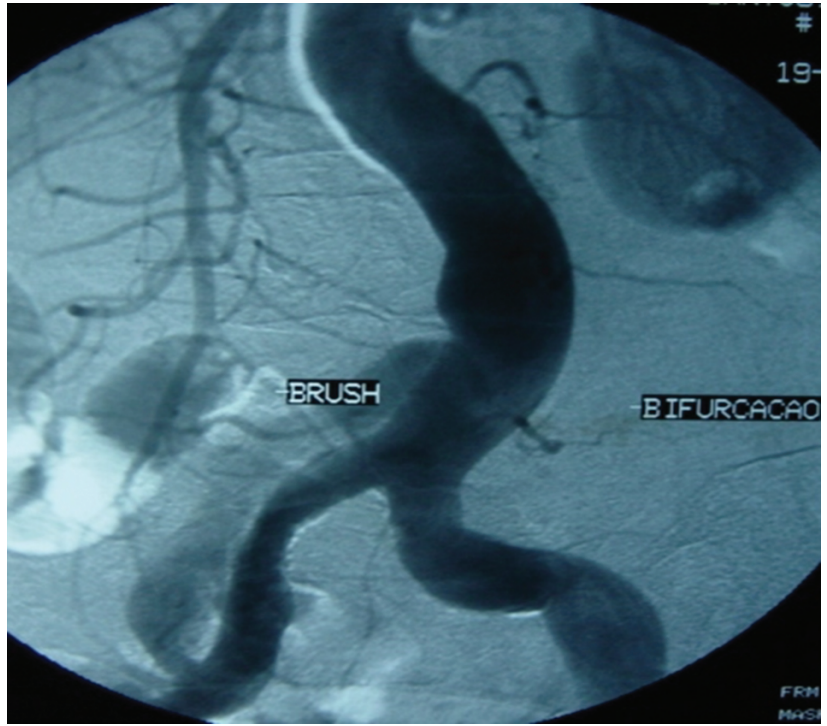

Figura 12. Arteriografia: dissecção aorto-ilíaca (caso 2). 
O orifício de entrada nas dissecções espontâneas da aorta abdominal infrarrenal ocorre, mais frequentemente, entre a artéria renal e a artéria mesentérica inferior e, em cerca de metade dos casos, a extensão distal da dissecção restringe-se ao segmento aórtico ${ }^{6}$.

Dor abdominal aguda é o sintoma mais comum presente nos casos de DEAAI, mas um número significativo de pacientes é assintomático ${ }^{7}$. Num estudo com 10 pacientes com $\mathrm{DEAAI}^{6}$ a dor abdominal foi notada em 7 pacientes. Um paciente tinha dor de repouso em membros inferiores e 2 pacientes eram assintomáticos. Três pacientes tinham história de febre baixa. Em 3 pacientes foi notada uma massa abdominal palpável.

O diagnóstico da dissecção aórtica requer um alto índice de suspeição. Elementos do passado clínico como hipertensão, presença de doença aneurismática da aorta ou história familiar de doenças do tecido conjuntivo são freqüentes fatores de risco e podem ajudar a estabelecer o diagnóstico ${ }^{10}$.

Atualmente o diagnóstico da DEAAI baseia-se no ultra-som, na TC de abdome, na angiografia e na MRI ${ }^{6}$. No estudo de Farber e cols ${ }^{6}$, o diagnóstico foi feito com TC abdominal em 8 pacientes, com arteriografia em um paciente e com cirurgia em um paciente. Acredita-se que antes de submeter o paciente a um procedimento cirúrgico de grande porte como a substituição da aorta abdominal por próteses, informações mais precisas podem ser obtidas por TC de abdome com contraste e com angiografia e alguns autores consideram esta combinação mandatória ${ }^{11}$.

Em número significativo de pacientes (40\%), a DEAAI evolui com formação de aneurismas. A dissecção crônica pode evoluir com degeneração aneurismática por fraqueza da parede aórtica e estar associada a maior risco de rotura. Geralmente, a rotura ocorre na junção da dissecção e da parede da aorta normal e, nessa eventualidade, o risco de óbito eleva-se, chegando a $90 \%{ }^{9}$.

A história natural da DEAAI não está estabelecida devido à raridade da condição. $\mathrm{O}$ risco do tratamento na DEAAI é significativamente menor do que na dissecção de aorta torácica descendente. Razões para intervenção nos pacientes com DEAAI citadas na literatura incluem ruptura aórtica, isquemia de extremidades inferiores, dor intratável, associação com aneurisma aórtico e prevenção de degeneração aneurismática futura ${ }^{6,9}$.

As opções terapêuticas nos casos de DEAAI incluem substituição protética da aorta envolvida ou tratamento clínico ${ }^{5}$. Existem relatos isolados de tratamento endovascular ${ }^{3,6,12,13} \mathrm{e}$, em alguns casos, guiado por ultra-sonografia intravascular ${ }^{13}$.

A terapia conservadora em pacientes com DEAAI inclui medicação anti-hipertensiva, se necessário, e seguimento cuidadoso ${ }^{6}$.
O tratamento de escolha das DEAAI com as complicações descritas acima é o cirúrgico convencional, que consiste em substituir a aorta e/ou segmento ilíaco por prótese de Dacron tubular ou bifurcada9.

Pacientes com isquemia de extremidades inferiores podem ser tratados tanto com cirurgia convencional como por técnicas endovasculares. A decisão deve se basear no risco operatório, dimensões arteriais e morfologia arterial ${ }^{6}$. O tratamento endovascular permanece um desafio nos casos agudos em função do calibre da aorta abdominal, da extensão da dissecção para as ilíacas e da compressão da luz verdadeira pela luz falsa ${ }^{2}$. Os pacientes que evoluem para formação aneurismática devem ser operados em função do risco de rotura ${ }^{6}$.

A dissecção espontânea da aorta abdominal infrarrenal (não associada a trauma ou a dissecção de aorta torácica descendente) é rara. Entretanto, o uso disseminado de ultra-som abdominal e de TC de abdome nos casos de dor abdominal inespecífica aumentou consideravelmente o diagnóstico desta condição $0^{5}$. Esta entidade pode evoluir com ruptura aguda em $17 \%$ dos casos, com elevada mortalidade, portanto é importante o diagnóstico correto e precoce, além da adoção de medidas clínicas ou cirúrgicas necessárias em cada caso.Em nossos casos relatados os diagnósticos foram baseados no quadro clínico de dor abdominal súbita e confirmada a hipótese diagnøstica em exames complementares como ultrassom abdominal, aortografia e angiotomografia. As pacientes foram submetidas a tratamento cirúrgico por apresentarem complicações. Uma das pacientes foi submetida a tratamento de escolha nos casos de DEAAI que e a revascularização com prótese de Dacron por apresentar dissecção extensa da aorta distal ate ilíaca comum direita. No outro caso optamos por cirurgia não convencional em casos de DEAAI de endarterectomia da placa dissecada, com fixação distal, pelo fato da paciente apresentar dissecção segmentar de apenas $2 \mathrm{~cm}$ e os segmentos aórticos proximais e distais apresentarem bom aspecto, ao invés de cirurgia de revascularização com prótese .

Acreditamos ser hoje o tratamento endovascular uma ótima opção em casos de DEAAI apesar de o tratamento de escolha ser a revascularização convencional com prótese de Dacron.

O tratamento conservador com drogas anti-hipertensivas e acompanhamento cuidadoso com exames de imagem incorrem em risco de degeneração aneurismática futura. Acreditamos também que o tratamento cirúrgico de endarterectomia da placa dissecada, desde que em segmento restrito, possa ser uma opção bem viável principalmente em serviços de saúde pública em que não haja o credenciamento de tratamento endovascular. 


\section{Referências}

1. Anacleto A, Anacleto JC. Dissecção aórtica. In: Maffei FHA, Lastória S, Yoshida WB, Rollo HA. Doenças vasculares periféricas. Rio de Janeiro: MEDSl; 2002. p. 1173-214.

2. Barros Junior N, Amorim JE, Perez MCJ, Miranda Junior F. Spontaneous infrarenal abdominal aortic dissection: case report and literature review. J Vasc Bras. 2004;3(4):379-82.

3. Mózes G, Gloviczki P, Park WM, Schultz HL, Andrews JC. Spontaneous dissection of infrarenal abdominal aorta. Semin Vasc Surg. 2002;15(2):128-36. http://dx.doi.org/10.1053/svas.2002.33094

4. Porcellini $M$, Mainenti $P$, Bracale U. Endograft repair of spontaneous infrarenal abdominal aortic dissection. J Vasc Surg. 2005;41(1):155. http://dx.doi.org/10.1016/j.jvs.2004.01.040

5. Borioni R, Garofalo M, De Paulis R, Nardi P, Scaffa R, Chiarello L. Abdominal Aortic dissections: anatomic and clinical features and therapeutic options. Tex Heart Inst J. 2005;32(1):70-3. PMid:15902826.

6. Farber $\mathrm{A}, \mathrm{Wagner} \mathrm{WH}, \mathrm{Cossman} \mathrm{DV}$, et al. Isolated dissection of abdominal aorta: clinical presentation and therapeutic options. I Vasc Surg. 2002;36(2):205-10. http://dx.doi.org/10.1067/mva.2002.125028

7. Trimarchi S, Tsai T, Eagle KA, et al. Acute abdominal aortic dissection: insight from the International Registry of Acute Aortic Dissection (IRAD). J Vasc Surg. 2007;46(5):913-9. http://dx.doi.org/10.1016/j. jvs.2007.07.030

8. Mantelas M, Antonitsis P, Kaitzis D, Hatzibaloglou A, Moros I. Spontaneous isolated dissection of the abdominal aorta: single-center experience. Interact Cardiovasc Thorac Surg. 2009;8(4):398-401. http://dx.doi.org/10.1510/icvts.2008.198051

9. Farber A, Lauterbach SR, Wagner WH, et al. Spontaneous infrarenal abdominal aortic dissection presenting as claudication: case report and review of the literature. Ann Vasc Surg. 2004;18(1):4-10. PMid:14712378

10. Green GR, Kron IL. Aortic dissection. In: Cohn LH, Edmunds Junior LH, editors. Cardiac surgery in the adult. New York: McGrawHill; 2003. p. 1095-122.

11. Kibria SM, Leveson SH, Homer-Vanniasinkam S. Spontaneous dissection of abdominal aorta. J R Coll Surg Edinb. 2000;45(6):408-10.

12. Berthet JP, Marty-Ané $\mathrm{CH}$, Veerapen R, Picard E, Mary H, Alric P. Dissection of abdominal aorta in blunt trauma: endovascular or conventional surgical management? J Vasc Surg. 2003;38(5):997-1003. http://dx.doi.org/10.1016/S0741-5214(03)00613-X

13. Giudice R, Frezzoti A, Scoccianti M. Intravascular ultrasound-guided stenting for chronic abdominal aortic dissection. J Endovasc Ther. 2002;9(6):926-31. PMid:12546599.

Correspondência Otacílio de Camargo Junior Rua Cândido Gomide, 468, Jardim Guanabara, CEP 13073-200, Campinas (SP) Brasil E-mail: otacamjr@terra.com.br

Contribuições dos autores: Concepção e desenho do estudo: OC), SAG, MFM Análise e interpretação dos dados: OC), GCGA, MFM, Coleta de dados: KCM, MVF, OC Redação do artigo:KCM,MFM, OC) Revisão crítica do texto:OC), MPA, GBM Aprovação final do artigo*: OCJ,MPA, GCGA, GBM Análise estatística: $O C$ Responsabilidade geral pelo estudo: OC] *Todos os autores leram e aprovaram a versão final submetida ao I Vasc Bras. 